\title{
Multiplex testing for Factor II and Factor V mutations in thrombophilia: technical verification and clinical validation of the $\operatorname{cobas}^{\circledR}$ Factor II and Factor V test
}

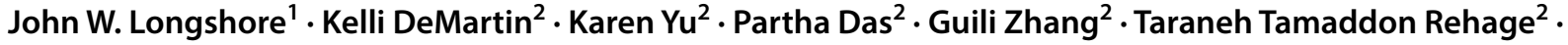 \\ Deepa Jethwaney ${ }^{2} \cdot$ Sylwia Karwowska² ${ }^{2}$
}

Published online: 3 October 2018

(C) The Author(s) 2018

\begin{abstract}
Laboratory testing for thrombophilia is complicated but essential for diagnosis. In 2017, the cobas ${ }^{\circledR}$ Factor II and Factor V Test (cobas F2F5 test) was launched for use with the cobas $\mathbf{z} 480$ analyzer. This qualitative polymerase chain reaction test enables multiplex Factor II and Factor V testing with flexible reporting and workflow efficiency. Here, we report the results from studies investigating the performance of the cobas F2F5 test. Technical performance verification, clinical validation, external laboratory performance, and workflow comparison studies were performed. Fresh and frozen whole-blood and genomic DNA (gDNA) samples were tested, and several manual and automated DNA isolation methods were used. Bidirectional Sanger sequencing was used to verify genotypes identified by the cobas F2F5 test. One hundred percent agreement between the cobas F2F5 test and Sanger sequencing was observed for all genotypes. An external laboratory using remnant clinical samples also yielded 100\% agreement between cobas F2F5 test results and their routine testing method. The cobas F2F5 test reduced the total sample processing time compared with the LightCycler® 1.2 platform (98.6 vs 420.2 min; 96 samples). Hemoglobin, extraction buffer, and ethanol contamination of the gDNA sample can lead to invalid results. The cobas F2F5 test has a high degree of accuracy for identification of Factor II and Factor V genotypes. This multiplex testing with short sample processing time can reduce handling errors and increase efficiency. Both manual and automated DNA isolation methods can be used with the cobas F2F5 test.
\end{abstract}

Keywords Thrombophilia $\cdot$ cobas ${ }^{\circledR}$ Factor II and Factor V test · Factor II G20210A · Factor V Leiden

\section{Key messages}

- Roche Molecular Systems recently launched a new realtime polymerase chain reaction test, the cobas ${ }^{\circledR}$ Factor II and Factor V Test, to detect common mutations associated with thrombophilia [13]

Electronic supplementary material The online version of this article (https://doi.org/10.1007/s11239-018-1745-8) contains supplementary material, which is available to authorized users.

Sylwia Karwowska

sylwia.karwowska@ roche.com

1 Carolinas Pathology Group and Carolinas HealthCare System, Charlotte, NC, USA

2 Roche Molecular Systems Inc, 4300 Hacienda Drive, Pleasanton, CA 94588, USA
- The cobas F2F5 test allows for multiplex testing with flexible reporting and a user-selected sample DNA extraction method

- Technical performance and clinical validation studies found $100 \%$ agreement between genotypes reported by the cobas F2F5 test and reference Sanger sequencing

- Compared with the LightCycler® 1.2 platform-based testing method, the cobas F2F5 test also reduces the total processing time and number of steps required in amplification and detection

- These results show that the new cobas F2F5 test is both time saving and cost-efficient and provides a high level of accuracy in Factor II and Factor V genotype identification 


\section{Introduction}

Thrombophilia, characterized by a predisposition to the development of thrombi in veins, arteries, or both, is the result of either inherited or acquired defects (or an interaction between the 2) in the coagulation system [1, 2]. The most commonly associated genetic mutations for inherited thrombophilia are gain-of-function mutations in the genes for Factor V and Factor II (prothrombin). The American College of Medical Genetics and recent guidelines from other professional societies recommend genetic testing only in certain circumstances for patients with suspected hereditary venous thrombosis [3, 4]. Patients who test positive for Factor V Leiden should be considered for Factor II G20210A testing, particularly pregnant women with a previous history of venous thromboembolism and recurrent pregnancy loss [3-5].

Several different laboratory testing methods are currently available for Factor II and Factor V mutation detection [6]. Based on internal data from market assessments and sales, we estimate that approximately 2.2 million Factor II and Factor $\mathrm{V}$ genotyping tests are performed annually worldwide (excluding Japan) [7]. The laboratory costs of thrombophilia testing are estimated to exceed $\$ 650$ million (USD) annually [8]. Therefore, there is an urgent need to optimize screening and diagnosis algorithms, make testing more cost-effective for laboratories, and reduce the frequency of inappropriate testing $[9,10]$.

In 2003, Roche Molecular Diagnostics launched 2 qualitative real-time polymerase chain reaction (PCR)-based diagnostic tests for use with the LightCycler@ 1.2 Instrument-the Factor II G20210A Kit and the Factor V Leiden Kit - each allowing for detection and genotyping of a single point mutation from whole-blood genomic DNA (gDNA) $[11,12]$. In 2017 , keeping with evolving laboratory practices and technological advances, the cobas ${ }^{\circledR}$ Factor II and Factor $\mathrm{V}$ Test (cobas F2F5 test) was launched for use with the cobas z 480 analyzer. This test not only allows for multiplex testing but also flexible reporting-either both mutations together or individually-depending on testing requirements. The testing algorithm was optimized to distinguish between wild-type and mutant alleles and designed to prevent the occurrence of incorrect results. For greater flexibility prior to amplification and detection, DNA extraction from whole blood can be performed offline using a user-selected manual
Fig. 1 Comparison of the 2 Roche Molecular Systems qualitative polymerase chain reaction (qPCR) methods for Factor II and Factor $\mathrm{V}$ detection using the LightCycler and cobas z 480 instruments. FII Factor II, FV Factor $\mathrm{V}, K_{2} E D T A$ dipotassium ethylenediaminetetraacetic acid, LIS laboratory information system

Fig. 2 Overall workflow for Factor II and Factor V identification using the cobas $\mathbf{z} 480$ platform. $K_{2} E D T A$ dipotassium ethylenediaminetetraacetic acid

\begin{tabular}{|c|c|c|}
\hline & $\begin{array}{l}\text { LightCycler }{ }^{2} \\
\text { Factor II and } \\
\text { Factor } V \\
\text { Tests }\end{array}$ & $\left\{\begin{array}{l}\text { cobas }^{*} \\
\text { Factor II and } \\
\text { Factor } \mathrm{V} \\
\text { Test }\end{array}\right.$ \\
\hline Sample Type & \multicolumn{2}{|c|}{ K2EDTA whole blood specimens } \\
\hline Intended Use & \multicolumn{2}{|c|}{ aid in diagnosis of patients with suspected thrombophilia } \\
\hline Batch Size Options (in patient samples) & 15 or 30 & $1-94$ \\
\hline LIS Result Reporting & No & Yes \\
\hline User-Selected Sample Preparation & MagNA Pure LC or High Pure & User-Selected \\
\hline Result & Individual tests for Fll and FV & Single-Well Multiplex Test for both FII \& FV \\
\hline Patient Samples Per Kit & 30 & 96 \\
\hline
\end{tabular}

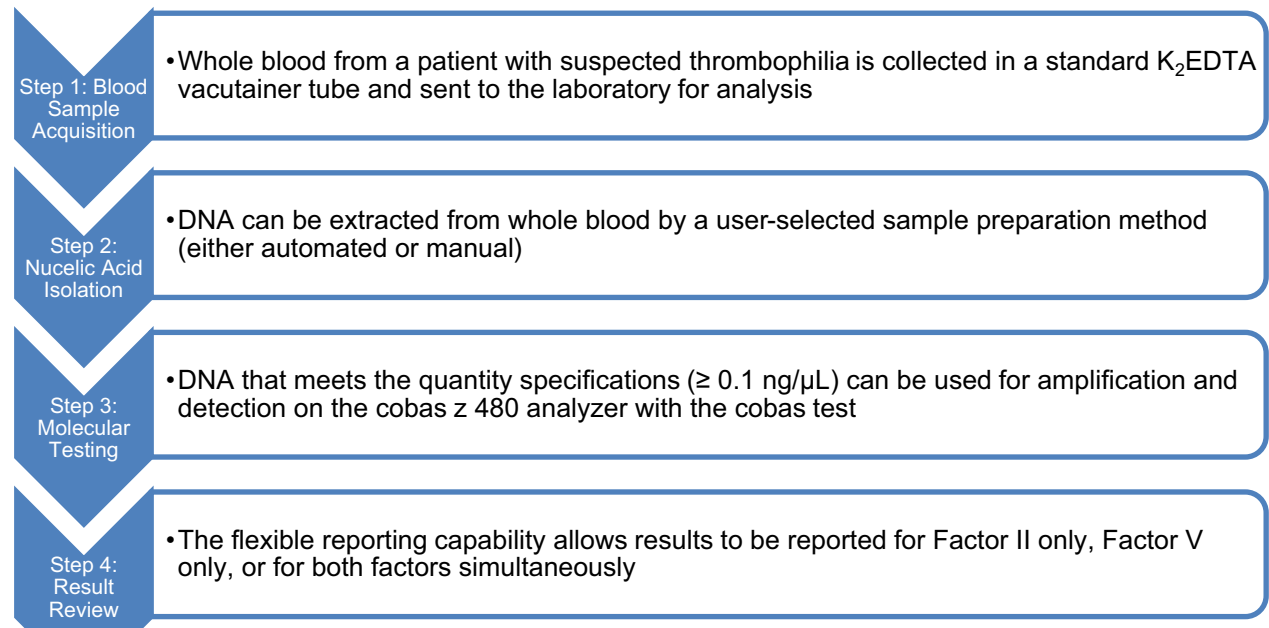


or automated method. Multiplex testing also improves costefficiency by reducing the testing time and minimizing the risk of error. Figure 1 summarizes the differences between the earlier LightCycler and the new cobas $\mathbf{z} 480$ platforms.

Here, we report the results from technical performance verification, clinical validation, and external laboratory performance studies for the cobas F2F5 test.

\section{Methods}

The overall workflow for Factor II and Factor V testing using the cobas F2F5 test platform is summarized in Fig. 2.

\section{Technical performance verification}

As part of the development of the cobas F2F5 test, studies were designed to investigate the performance characteristics of the test, such as the limit of detection, analytical specificity, stability (including samples and reagents), and substances that may interfere with the performance of the test. Further details about the studies are provided in Supplementary Table 1.

\section{Clinical validation studies}

\section{Method comparison}

Frozen whole-blood and DNA specimens $(n=300)$ were obtained from laboratories that performed Factor II and/or Factor V testing per routine medical care. Genotypes tested included Factor II or Factor V homozygous mutations, single or compound heterozygous mutations, and wild-type samples. Factor II homozygous mutant samples $(n=16)$ were available as gDNA only. Manual gDNA isolation using the High Pure (HP) PCR Template Preparation Kit (Roche Molecular Systems Inc, Pleasanton, CA) and cobas F2F5 testing were carried out at an external site. Bidirectional
Sanger DNA sequencing was carried out at a commercial laboratory.

\section{Clinical reproducibility}

Nine panel members were tested at three sites, with each site using a different commercially available manual DNA extraction kit: HP kit (Roche Molecular Systems Inc, Pleasanton, CA), ReliaPrep ${ }^{\mathrm{TM}}$ Blood gDNA Miniprep System (Promega, Madison, WI), and QIAamp® DSP DNA Blood Mini Kit (Qiagen, Venlo, the Netherlands). The nine panel members tested consisted of four dipotassium ethylenediaminetetraacetic acid $\left(\mathrm{K}_{2}\right.$ EDTA) whole-blood samples, three contrived whole-blood samples, and two gDNA samples (diluted to $0.2 \mathrm{ng} / \mu \mathrm{L}$ ). Each testing site had two operators and one instrument. Each operator performed 1 run per day over 5 non-consecutive days. A total of 18 cobas F2F5 tests were carried out for each panel member (in duplicate) for each reagent lot.

\section{External laboratory performance testing}

Performance of the cobas F2F5 test in combination with automated DNA extraction methods was assessed using frozen and fresh clinical samples. Samples were de-identified prior to processing and testing. Banked (frozen) whole-blood specimens $(n=200)$-a subpopulation of samples used for the method comparison clinical validation study (using manual DNA isolation; outlined above)were provided by Roche Molecular Systems. gDNA was isolated using both the MagNA Pure 24 (MP 24) and MagNA Pure 96 (MP 96) automated DNA isolation methods. Overall percentage agreement (OPA) was assessed against archived Sanger sequencing results and between DNA isolation methods. Remnant clinical samples (fresh, never frozen; $n=200$ ) from routine testing were provided by an external study site and DNA was isolated using the MP 96 isolation method. The cobas F2F5 test results were compared with results from routine Factor II and Factor V
Fig. 3 Overview of the workflow using the MP 96 and cobas z 480 platform for the $\operatorname{cobas}{ }^{\circledR}$ Factor II and Factor V Test. MP 96, MagNA Pure System

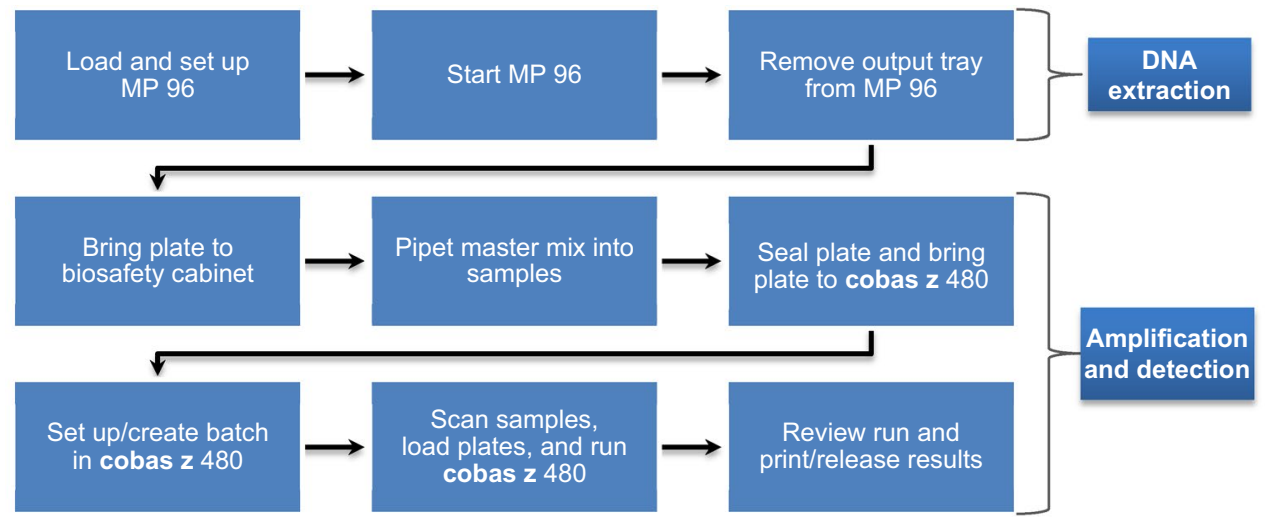


testing methods used at the external site (Roche Factor II prothrombin G20210A kit and Roche Factor V Leiden Kit coupled to the LightCycler ${ }^{\circledR} 1.2$ instrument).

\section{Workflow comparison}

The workflow for cobas Factor II and Factor V testing using the cobas $\mathbf{z} 480$ platform was compared with the LightCycler® 1.2 platform. The hands-on and total processing times in addition to the number of steps from specimen receipt to release of results were observed and documented for different batch sizes. DNA isolation was carried out using the MP 96 instrument prior to testing with both platforms. Figure 3 shows representative steps in the workflow comparison study using the example of the MP 96 instrument paired with the cobas z 480 for the new cobas F2F5 test (MP 96 paired with LightCycler steps not shown).

\section{Study ethics}

All studies were conducted in compliance with their protocols. Clinical studies were conducted with the International Conference on Harmonisation Good Clinical Practice Guidelines and regulations of the US Food and Drug Administration (FDA). Where appropriate, study protocols were submitted to an institutional review board in accordance with FDA and local regulatory requirements prior to the start of the study.

\section{Results}

\section{Technical performance verification studies}

All studies met predefined (prior to test development) acceptance criteria. The aims and key results from each technical performance study are outlined in Table 1.

\section{Clinical validation studies}

\section{Method comparison}

All runs and sample results were valid (no repeat tests were needed). A genotype result was classified as correct if the same genotype was detected by both the cobas F2F5 test and the reference method, bidirectional Sanger sequencing.

For Factor II testing $(\mathrm{n}=300)$, the OPA with bidirectional Sanger sequencing was $100 \%$, with a 2 -sided $95 \%$ lower confidence boundary (LCB; exact method) of $98.78 \%$. The negative percentage agreement (NPA; for wild-type samples) was $100 \%(95 \% \mathrm{LCB}, 97.55 \%$; $\mathrm{n}=149)$. The positive percentage agreement (PPA; for Factor II mutation-positive samples) was also $100 \%$ (95\% LCB, $97.59 \%$; $n=151)$. Of the 151 positive samples, the percentage agreement for both heterozygous and homozygous mutant samples was $100 \%$ each (95\% LCB, 97.20\%; $\mathrm{n}=130$ and 95\% LCB, 83.89\%; $\mathrm{n}=21$, respectively).

Similarly, for Factor V testing, the OPA was 100\% (95\% LCB, 98.78\%; $n=300$ ), NPA was $100 \%$ (95\% LCB, $97.52 \%$; $\mathrm{n}=147)$, and PPA was $100 \%$ (95\% LCB, 97.62\%; $\mathrm{n}=153)$. For the positive samples, the percentage agreement for both heterozygous and homozygous mutant samples was $100 \%$ each $(95 \%$ LCB, $97.20 \% ; \mathrm{n}=130$ and $95 \% \mathrm{LCB}, 85.18 \%$; $\mathrm{n}=23$, respectively).

\section{Clinical reproducibility}

Four hundred twenty whole-blood samples (240 clinical and 180 contrived) and 120 gDNA samples were tested. A total of 540 tests obtained from 30 valid runs yielded 539 valid results. One invalid result was due to a lack of agreement in the data parameter checks for software result interpretation, designed to prevent inaccurate result reporting. The invalid rate for whole-blood samples in the study was $0.24 \%$ ( 1 of 420).

The cobas F2F5 test demonstrated $100 \%$ correct call rates for each Factor II and Factor V genotype tested (Table 2).

\section{External laboratory performance testing}

For banked (frozen) specimens, the cobas F2F5 test results agreed with the genotypes determined by Sanger sequencing for $100 \%$ of all genotypes tested regardless of the DNA isolation method used, manual or automated $(n=200 ; 95 \%$ LCB, 98.2\%). For Factor II genotypes, the percentage agreement was $100 \%$ each for wild-type $(n=103)$, heterozygous mutant $(n=94)$, and homozygous mutant $(n=3)$ samples. For Factor $\mathrm{V}$ genotypes, the percentage agreement was also $100 \%$ each for wild-type $(n=87)$, heterozygous mutant $(\mathrm{n}=96)$, and homozygous mutant $(\mathrm{n}=17)$ samples.

Results from remnant whole-blood specimens tested with the MP 96 platform showed that the cobas F2F5 test generated $100 \%$ correct calls and 100\% OPA compared with the LightCycler method for all Factor II and Factor V genotypes tested (95\% LCB, 98.17\%; $\mathrm{n}=200$ ). For Factor II genotypes, the percentage agreement was $100 \%$ for both wild-type $(n=196)$ and heterozygous mutant $(n=4)$ samples (no homozygous mutant samples were tested). Similarly, for Factor V genotypes, the percentage agreement was $100 \%$ each for wild-type $(n=178)$, heterozygous mutant $(n=21)$, and homozygous mutant $(\mathrm{n}=1)$ samples. 
Table 1 Summary of results from technical performance verification studies for the cobas F2F5 test

\begin{tabular}{lll}
\hline Study type Name Aim and key findings & Ne
\end{tabular}

DNA isolation

DNA extraction method study

Specificity

Target sequence exclusivity study

Interference and contamination Potentially interfering substances study

Potentially interfering mutations study

Cross-contamination study

Stability

Whole-blood stability study
Aim To determine the performance of the cobas F2F5 test on gDNA isolated from $\mathrm{K}_{2}$ EDTA whole blood using 3 different commercially available kits

Result For each DNA isolation method, the cobas F2F5

test yielded $100 \%$ correct results when compared with bidirectional Sanger sequencing

One of the gDNA samples isolated was rust colored and yielded invalid results (see Discussion)

Aim To determine the exclusivity of the cobas F2F5 test for the Factor II prothrombin and Factor V Leiden mutations and wild-type alleles

Result BLAST searching of the EMBL nucleotide sequence database and human genome did not identify any sequences with the potential to cross-react or interfere with the cobas F2F5 test

Aim To determine the impact of potentially interfering substances present in whole blood or gDNA on the performance of the cobas F2F5 test

Result Correct genotype results were obtained for 6 whole-blood specimens that were tested with a panel of interfering substances

Ethanol (5\% v/v) and extraction buffer $(2.5 \% \mathrm{v} / \mathrm{v})$ added to gDNA interfered with the cobas F2F5 test and caused invalid results (see Discussion)

Current standard-of-care blood-thinner drugs (eg, heparin, warfarin, rivaroxaban, dabigatran etexilate) did not interfere with the test results

Aim To determine the effect of known SNPs close to the Factor II 20210 or Factor V 1691 locus on the detection of the Factor II $21210 \mathrm{G}>\mathrm{A}$ and Factor V $1691 \mathrm{G}>$ A mutations using the cobas F2F5 test

Result None of the SNP plasmids caused false-positive results. All but one of the SNPs were detected as wild-type. The Factor V 1689G > A mutation was not detected by the cobas F2F5 test when present on both alleles (see Discussion)

Aim To determine the frequency of cross-contamination during manual gDNA isolation and execution of the cobas F2F5 test

Result The cross-contamination rate was $0 \%$

Aim To determine the stability of $\mathrm{K}_{2}$ EDTA wholeblood specimens

To compare the performance of the cobas F2F5 test on gDNA isolated from freshly drawn whole-blood samples vs whole blood that was frozen and thawed up to three times

Result Correct results were obtained for all samples, storage conditions, and time intervals tested, including up to 3 freeze/thaw cycles at $-20{ }^{\circ} \mathrm{C}$ and $\leq-70{ }^{\circ} \mathrm{C}$ for whole blood

$\mathrm{K}_{2}$ EDTA whole-blood samples may be stored at $2{ }^{\circ} \mathrm{C}-8{ }^{\circ} \mathrm{C}$ for up to 7 days, $25^{\circ} \mathrm{C}-30{ }^{\circ} \mathrm{C}$ for up to 3 days, or frozen at $-20^{\circ} \mathrm{C}$ and $\leq-70^{\circ} \mathrm{C}$ for up to 8 months 
Table 1 (continued)

\begin{tabular}{ll}
\hline Study type & Name \\
\hline & gDNA stability study
\end{tabular}

gDNA stability study

Open reagent stability study

Activated MMX stability study

Activated MMX plus extracted sample stability study

Analytical sensitivity and

DNA concentration
Lower limit of analytical sensitivity

Upper limit of analytical sensitivity

Validation of DNA concentration from whole-blood specimens
Aim and key findings

Aim To determine the stability of gDNA isolated from $\mathrm{K}_{2}$ EDTA whole-blood specimens

To compare the performance of the cobas F2F5 test on gDNA (isolated using three commercially available sample preparation methods) from freshly drawn whole-blood samples vs frozen whole blood that was thawed up to three times

Result Correct results were obtained for all samples, storage conditions, sample preparation methods, and time intervals tested, including up to 3 freeze/thaw cycles at $-20{ }^{\circ} \mathrm{C}$ and $\leq-70{ }^{\circ} \mathrm{C}$ for gDNA

gDNA isolated from $\mathrm{K}_{2}$ EDTA whole-blood samples may be stored at $2{ }^{\circ} \mathrm{C}-8{ }^{\circ} \mathrm{C}$ for up to 7 days, or frozen at $-20{ }^{\circ} \mathrm{C}$ and $\leq-70{ }^{\circ} \mathrm{C}$ for up to 12 months

Aim To determine the stability of opened reagents (including controls) of the cobas F2F5 test after they have been used once

Result Kit reagents are stable at $2{ }^{\circ} \mathrm{C}-8{ }^{\circ} \mathrm{C}$ for at least 90 days for up to two uses (only two uses were tested)

Aim To determine the stability of activated MMX at room temperature (MMX is activated when combined with the cofactor)

Result Activated MMX stability acceptance criteria were met at all tested time intervals. Activated MMX may be stored at room temperature for at least $4 \mathrm{~h}$

Aim To determine the stability of activated MMX combined with processed specimens (gDNA isolated from whole blood) at room temperature (up to $30^{\circ} \mathrm{C}$ )

Result All tests of all samples yielded correct results when compared with bidirectional Sanger sequencing. Activated MMX with isolated DNA or run controls is stable for at least $2 \mathrm{~h}$ at room temperature

Aim To determine the limit of detection and minimum input gDNA concentration for the cobas F2F5 test

Result The lowest input gDNA concentration with at least $\geq 95 \%$ correct genotype results was $0.01 \mathrm{ng} / \mu \mathrm{L}$

The recommended lowest gDNA input concentration is $0.1 \mathrm{ng} / \mu \mathrm{L}$ (see Discussion)

Aim To determine the maximum concentration of gDNA input for the cobas F2F5 test

Result Correct results were observed with gDNA input as high as $300 \mathrm{ng} / \mu \mathrm{L}$. The recommended highest gDNA input concentration is $150 \mathrm{ng} / \mu \mathrm{L}$ (see Discussion)

Aim To validate the recommended quantity and quality specifications for gDNA from $>300 \mathrm{~K}_{2}$ EDTA whole blood as determined by UV spectroscopy after isolation using various commercially available DNA isolation methods

Result cobas F2F5 test results agreed with Sanger sequencing for all samples that met the following specifications

$95 \%$ of gDNA samples had a DNA concentration $\geq$ $2 \mathrm{ng} / \mu \mathrm{L}$ and $99 \%$ of gDNA samples had a DNA concentration $\geq 0.2 \mathrm{ng} / \mu \mathrm{L}$

BLAST basic local alignment search tool, cobas F2F5 test cobas ${ }^{\circledR}$ Factor II and Factor V test, EMBL European Molecular Biology Laboratory, gDNA genomic DNA, $K_{2} E D T A$ dipotassium ethylenediaminetetraacetic acid, $M M X$ master mix, $S N P$ single-nucleotide polymorphism 
Table 2 Summary of results from the reproducibility study by site, lot, sample preparation method, and genotype for Factor II and Factor V testing using the cobas ${ }^{\circledR}$ Factor II and Factor V Test

\begin{tabular}{|c|c|c|c|c|}
\hline Site/lot/sample preparation method & Genotype panel & $\begin{array}{l}\text { Total sam- } \\
\text { ples tested }\end{array}$ & Correct calls & $\begin{array}{l}\text { Percentage agree- } \\
\text { ment }\left(95 \% \mathrm{LCB}^{\mathrm{a}}\right)\end{array}$ \\
\hline \multicolumn{5}{|l|}{ Reproducibility study for Factor II } \\
\hline \multirow[t]{3}{*}{ Site 1/Lot 1/Roche High Pure PCR Template Preparation Kit } & WT & 100 & 100 & $100.0(97.05)$ \\
\hline & HET & 60 & 60 & $100.0(95.13)$ \\
\hline & MUT & 20 & 20 & $100.0(86.09)$ \\
\hline \multirow[t]{3}{*}{ Site 2/Lot 2/Promega ReliaPrep ${ }^{\mathrm{TM}}$ Blood gDNA Miniprep System } & WT & 100 & 100 & $100.0(97.05)$ \\
\hline & HET & 60 & $59^{\mathrm{b}}$ & $100.0(95.05)$ \\
\hline & MUT & 20 & 20 & $100.0(86.09)$ \\
\hline \multirow[t]{3}{*}{ Site 3/Lot 3/Qiagen QIAamp® DSP DNA Blood Mini Kit } & WT & 100 & 100 & $100.0(97.05)$ \\
\hline & HET & 60 & 60 & $100.0(95.13)$ \\
\hline & MUT & 20 & 20 & $100.0(86.09)$ \\
\hline \multicolumn{5}{|l|}{ Reproducibility Study for Factor V } \\
\hline \multirow[t]{3}{*}{ Site $1 /$ lot $1 /$ Roche HIAGH pure PCR template preparation kit } & WT & 100 & 100 & $100.0(97.05)$ \\
\hline & HET & 60 & 60 & $100.0(95.13)$ \\
\hline & MUT & 20 & 20 & $100.0(86.09)$ \\
\hline \multirow[t]{3}{*}{ Site 2/Lot 2/Promega ReliaPrep Blood gDNA Miniprep System } & WT & 100 & 100 & $100.0(97.05)$ \\
\hline & HET & 60 & $59^{\mathrm{b}}$ & $100.0(95.05)$ \\
\hline & MUT & 20 & 20 & $100.0(86.09)$ \\
\hline \multirow[t]{3}{*}{ Site 3/Lot 3/Qiagen QIAamp DSP DNA Blood Mini Kit } & WT & 100 & 100 & $100.0(97.05)$ \\
\hline & HET & 60 & 60 & $100.0(95.13)$ \\
\hline & MUT & 20 & 20 & $100.0(86.09)$ \\
\hline
\end{tabular}

gDNA genomic DNA, HET heterozygous, LCB lower confidence bound, MUT homozygous mutant, WT wild-type

${ }^{a}$ Overall percentage agreement with $95 \%$ lower confidence bound of $98.17 \%$ (2-sided $95 \%$ LCB was calculated using the exact method)

${ }^{\mathrm{b}}$ Invalid result that was not retested

\section{Workflow overview study}

Results for the hands-on and total processing times in the pre-analytic (gDNA isolation) phase are provided in Supplementary Table 2.

Compared with the LightCycler ${ }^{\circledR}$ 1.2-based method, the cobas F2F5 test required less hands-on time (141.2 vs $8.6 \mathrm{~min}$ ), less total processing time (420.2 vs $98.6 \mathrm{~min}$ ), and fewer steps for amplification and detection (1091 vs 303; $\mathrm{n}=96$ samples). The processing times for 24 and 48 samples are provided in Supplementary Table 2.

\section{Discussion}

In this paper, we report results from several studies evaluating the performance characteristics of the new cobas F2F5 test for thrombophilia. In all, these studies show that the cobas F2F5 test is robust and has a very high degree of accuracy in determining Factor II and Factor V genotypes from frozen and fresh whole-blood and gDNA samples.
The minimum recommended gDNA input for the cobas F2F5 test is $0.1 \mathrm{ng} / \mu \mathrm{L}$, which is close to that observed for $>99 \%$ of gDNA samples used in our testing $(\geq 0.2 \mathrm{ng} / \mu \mathrm{L})$. However, correct results were generated at a concentration that is 10 times lower than recommended (Table 1). The maximum recommended gDNA concentration is $150 \mathrm{ng} /$ $\mu \mathrm{L}$, which is a conservative recommendation since correct results were generated using a concentration up to $300 \mathrm{ng} /$ $\mu \mathrm{L}$ (Table 1). No incorrect genotypes were reported in these studies.

One gDNA plasma sample with a rust color, most likely due to hemoglobin (a polymerase chain reaction inhibitor), yielded an invalid result in the DNA extraction method study (Table 1). It is recommended, therefore, that the appearance of gDNA isolated from whole blood should be clear and colorless; samples that contain a red-colored tint may yield invalid or incorrect results. Extraction buffers and ethanol in commercial DNA isolation kits can also cause invalid results (Table 1). These buffers typically contain chaotropic salts (eg, guanidine hydrochloride), which are potent denaturants and inhibit DNA polymerase. However, any residual extraction buffer is typically removed during the wash steps between lysis and elution in commercial DNA isolation kits. 
Ethanol is a common ingredient in the final wash buffer, and it is recommended that both the lysis and wash buffers are completely removed from the sample before running the cobas F2F5 test.

Eight known single-nucleotide polymorphisms close to the Factor II or Factor V locus were tested with the cobas F2F5 test and no false positives were detected. In the rare event that a Factor V $1689 \mathrm{G}>$ A mutation is present on both Factor V alleles, the cobas F2F5 test will yield an invalid result. All the aforementioned considerations are included in the cobas ${ }^{\circledR}$ Factor II and Factor V Test kit Instructions for Use.

In these studies, a variety of commercially available manual and automated DNA isolation methods were used, and the cobas F2F5 test identified Factor II and Factor V genotypes with a high degree of accuracy. The reproducibility study found $100 \%$ agreement between the cobas F2F5 test and Sanger sequencing for all genotypes and manual DNA isolation methods tested. Similarly, external laboratory performance testing found $100 \%$ agreement for all frozen and whole-blood specimens isolated using both manual and automated DNA extraction methods. These results highlight the "open front-end" capability of the cobas F2F5 test, which allows the user to select any gDNA isolation method, provided it meets the recommended minimum gDNA quantity [13].

Compared with the preceding LightCycler ${ }^{\circledR} 1.2$ platform, the cobas F2F5 test and cobas z 480 platform reduced the hands-on and total processing times for 96 samples by 16and fourfold, respectively. The total number of steps was also reduced by approximately fourfold, decreasing the likelihood of user processing errors.

Factor II and Factor V mutation testing is expensive, time consuming [7,10], and associated with errors that can lead to misdiagnosis or overtreatment [14]. The Italian Committee for the Standardization of Laboratory Tests reported a 4.9-17.0\% error rate for Factor V Leiden and 4.9-19.5\% error rate for Factor II G20210 identification [15]. The Royal College of Pathologists of Australasia reported a 98.63\% success rate for Factor V and Factor II mutation identification but found large variations between laboratories: $51 \%$ made $\geq 1$ error and 3 of 39 laboratories were responsible for nearly half the reported errors [16]. Results of genetic analyses can impact clinical decision making and patient levels of concern, thus testing laboratories should adhere to a high level of internal quality control and participate in external quality assurance programs [16].

In conclusion, the new cobas F2F5 test can improve the efficacy of thrombophilia testing through its established accuracy in genotype identification. In addition, the multiplex method and short processing time can reduce the cost and time associated with Factor II and Factor V testing. Lastly, the flexible reporting and "open front-end" design can enable easy integration into established testing procedures in laboratories.

Acknowledgements The authors would like to thank Steve Herman, Michelle Young (both of whom were employees of Roche Molecular Systems Inc, Pleasanton, California, USA at the time the research was conducted), and Shika Chug of Roche Molecular Systems Inc, Pleasanton, California, USA, for their support in operations, data management, and biostatistics. Medical writing support was provided by Malvika Katarya, Jonathan Lee, and Evelyn Rose of Health Interactions, Nucleus Global, San Francisco, California, USA.

Funding This study was sponsored by Roche Molecular Systems Inc, Pleasanton, California, USA.

\section{Compliance with ethical standards}

Conflict of interest Sylwia Karwowska, Kelli DeMartin, Karen Yu, Partha Das, Guili Zhang, Taraneh Tamaddon Rehage, and Deepa Jethwaney are employees of Roche Molecular Systems Inc. John W. Longshore has received research support from Roche Molecular Systems Inc.

Open Access This article is distributed under the terms of the Creative Commons Attribution 4.0 International License (http://creativeco mmons.org/licenses/by/4.0/), which permits unrestricted use, distribution, and reproduction in any medium, provided you give appropriate credit to the original author(s) and the source, provide a link to the Creative Commons license, and indicate if changes were made.

\section{References}

1. Dahlbäck B (2008) Advances in understanding pathogenic mechanisms of thrombophilic disorders. Blood 112:19-27. https://doi. org/10.1182/blood-2008-01-077909

2. Khan S, Dickerman JD (2006) Hereditary thrombophilia. Thromb J 4:15. https://doi.org/10.1186/1477-9560-4-15

3. Spector EB, Grody WW, Matteson CJ et al (2006) Technical standards and guidelines: venous thromboembolism (Factor V Leiden and prothrombin 20210G > A testing): a disease-specific supplement to the standards and guidelines for clinical genetics laboratories. https://www.acmg.net/pages/acmg_activities/stds2002/fv-pt.htm. Accessed June 18, 2018. https://doi.org/10.1007/ s11239-015-1316-1

4. Stevens SM, Woller SC, Bauer KA et al (2016) Guidance for the evaluation and treatment of hereditary and acquired thrombophilia. J Thromb Thrombolysis 41:154-164. https://doi. org/10.1007/s11239-015-1316-1

5. Connors JM (2017) Thrombophilia testing and venous thrombosis. N Engl J Med 377:1177-1187. https://doi.org/10.1056/NEJMr a1700365

6. Emadi A, Crim MT, Brotman DJ et al (2010) Analytic validity of genetic tests to identify factor $\mathrm{V}$ Leiden and prothrombin G20210A. Am J Hematol 85:264-270. https://doi.org/10.1002/ ajh. 21617

7. Roche Molecular Systems Inc. Data on file. 2016

8. Mou E, Kwang H, Hom J et al (2017) Magnitude of potentially inappropriate thrombophilia testing in the inpatient hospital setting. J Hosp Med 12:735-738. https://doi.org/10.12788/jhm.2819

9. Perez Botero J, Majerus JA, Strege AK, Johnson RD, Chen D, Pruthi RK (2017) Diagnostic testing approaches for activated 
protein $\mathrm{C}$ resistance and factor V Leiden: a comparison of institutional and national provider practices. Am J Clin Pathol 147:604610. https://doi.org/10.1093/ajcp/aqx033

10. Smith TW, Pi D, Hudoba M, Lee AY (2014) Reducing inpatient heritable thrombophilia testing using a clinical decision-making tool. J Clin Pathol 67:345-349. https://doi.org/10.1136/jclinpath2013-201840

11. Roche Molecular Diagnostics. Factor II prothrombin G20210A kit. https://molecular.roche.com/assays/factor-ii-prothrombi n-g20210a-kit/. Updated 2016. Accessed February 22, 2018

12. Roche Molecular Diagnostics. Factor V Leiden kit. https:// molecular.roche.com/assays/factor-v-leiden-kit/. Updated 2016. Accessed June 18, 2018

13. Roche Molecular Systems Inc (Updated 2017) cobas ${ }^{\circledR}$ Factor II and Factor V test, instructions for use. https://usdiagnostics.roche .com/download/en/document/technical/ms/08480575001-01.pdf. Accessed June 18, 2018

14. Favaloro EJ, McDonald D, Lippi G (2009) Laboratory investigation of thrombophilia: the good, the bad, and the ugly. Semin Thromb Hemost 35:695-710. https://doi. org/10.1055/s-0029-1242723

15. Tripodi A, Chantarangkul V, Menegatti M, Tagliabue L, Peyvandi F (2005) Performance of clinical laboratories for DNA analyses to detect thrombophilia mutations. Clin Chem 51:1310-1311. https ://doi.org/10.1373/clinchem.2005.049981

16. Hertzberg M, Neville S, McDonald D (2006) External quality assurance of molecular analysis of haemochromatosis gene mutations. J Clin Pathol 59:744-747. https://doi.org/10.1136/ jcp.2005.026005 